\title{
A Novel Vanadium Catalyzed Oxidation of Alcohols to Aldehydes and Ketones under Atmospheric Oxygen
}

\author{
Subbarayan Velusamy and T. Punniyamurthy ${ }^{\star}$ \\ Department of Chemistry, Indian Institute of Technology Guwahati, Guwahati 781039, India \\ Supporting Information
}

\section{Experimental Section}

General. Starting materials were purchased from Aldrich, Fluka and Lancaster and used without further treatment. $\mathrm{V}_{2} \mathrm{O}_{5}$ (99\% purity with yellow to rust brown powder) was obtained from $\mathrm{CDH}(\mathrm{P}) \mathrm{Ltd}$, Bombay. NMR spectra were recorded on DRX-300 (300 MHz for ${ }^{1} \mathrm{H}$ and 75.5 MHz for $\left.{ }^{13} \mathrm{C}\right)$ spectrophotometer using $\mathrm{CDCl}_{3}$ as a solvent and $\mathrm{Me}_{4} \mathrm{Si}$ as the internal standard. IR spectra were recorded on Nicolet impact 410 spectrophotometer. GC analysis was performed on HP-6890 series with a FID detector. Column chromatography was performed on silica gel (60-120 mesh) using ethyl acetate (or diethyl ether) and hexane as eluent.

Oxidation of Primary Alcohols. A mixture of primary alcohol $(2 \mathrm{mmol}), \mathrm{V}_{2} \mathrm{O}_{5}(0.018 \mathrm{~g}, 5 \mathrm{~mol} \%)$ and $\mathrm{K}_{2} \mathrm{CO}_{3}(1 \mathrm{mmol})$ was stirred in bluene $(3 \mathrm{ml})$ at $c a .100{ }^{\circ} \mathrm{C}$ for the appropriate time under atmospheric oxygen. After removal of the catalyst and $\mathrm{K}_{2} \mathrm{CO}_{3}$ by filtration, the filtrate was analyzed by $\mathrm{GC}$ and then passed through a short pad of silica gel using ethyl acetate and hexane as eluent or distilled to afford the analytically pure aldehydes.

Oxidation of Secondary Alcohols. The oxidation of secondary alcohols to ketones was performed as above in the absence of $\mathrm{K}_{2} \mathrm{CO}_{3}$.

All products were commercially available and identified by comparison of the isolated products with authentic samples.

Benzil. According to the general procedure benzoin gave $399 \mathrm{mg}(95 \%)$ of the product as a yellow solid after column chromatography (ethyl acetate/hexane=1:19). M.p. $95{ }^{\circ} \mathrm{C} ;{ }^{1} \mathrm{H}$ NMR $\left(\mathrm{CDCl}_{3}, 300 \mathrm{MHz}\right) \delta 7.8-8.0$ 
$(\mathrm{m}, 4 \mathrm{H}), 7.6-7.7(\mathrm{~m}, 2 \mathrm{H}), 7.3-7.5(\mathrm{~m}, 4 \mathrm{H}) ;{ }^{13} \mathrm{C} \mathrm{NMR}\left(\mathrm{CDCl}_{3}, 75.5 \mathrm{MHz}\right) \delta 195.1,135.4,133.6,130.4$, 129.6; IR (KBr) $1671 \mathrm{~cm}^{-1}$.

Benzyl benzoate. According to the general procedure benzyl alcohol gave $206 \mathrm{mg}$ (97\%) of the product as a colorless liquid after column chromatography (ethyl acetate/hexane=1:19). ${ }^{1} \mathrm{H} \mathrm{NMR}\left(\mathrm{CDCl}_{3}, 300 \mathrm{MHz}\right) \delta$ 8.0-8.2 (m, 2H), 7.3-7.6 (m, 8H), $5.4(\mathrm{~s}, 2 \mathrm{H}) ;{ }^{13} \mathrm{C} \mathrm{NMR}\left(\mathrm{CDCl}_{3}, 75.5 \mathrm{MHz}\right) \delta 166.3,136.0,132.9,130.1$, $129.6,128.5,128.3,128.2,128.1,66.6$; IR (neat) $1719 \mathrm{~cm}^{-1}$.

Benzaldehyde. The oxidation was performed as described in the general procedure using $540 \mathrm{mg}$ ( $5 \mathrm{mmol})$ of benzyl alcohol and the product was obtained $398 \mathrm{mg}(75 \%)$ as a colorless liquid after distillation. ${ }^{1} \mathrm{H}$ NMR $\left(\mathrm{CDCl}_{3}, 300 \mathrm{MHz}\right) \delta 10.2(\mathrm{~s}, 1 \mathrm{H}), 7.5-7.9(\mathrm{~m}, 5 \mathrm{H}) ;{ }^{13} \mathrm{C} \mathrm{NMR}\left(\mathrm{CDCl}_{3}, 75.5 \mathrm{MHz}\right) \delta 192.2,136.5,134.4$, 129.7, 128.9; IR (neat) $1695 \mathrm{~cm}^{-1}$.

4-Methoxybenzaldehyde. According to the general procedure 4-methoxybenzyl alcohol provided $250 \mathrm{mg}$ $(92 \%)$ of the product as a colorless liquid after chromatography (ethyl acetate/hexane=1:19). ${ }^{1} \mathrm{H}$ NMR $\left(\mathrm{CDCl}_{3}, 300 \mathrm{MHz}\right) \delta 9.9(\mathrm{~s}, 1 \mathrm{H}), 7.8(\mathrm{~d}, 2 \mathrm{H}, J=8.2 \mathrm{~Hz}), 7.0(\mathrm{~d}, 2 \mathrm{H}, J=8.2 \mathrm{~Hz}), 3.9(\mathrm{~s}, 3 \mathrm{H}) ;{ }^{13} \mathrm{C} \mathrm{NMR}$ $\left(\mathrm{CDCl}_{3}, 75.5 \mathrm{MHz}\right) \delta 190.6,164.6,131.9,130.0,114.3,55.5 ; \mathrm{IR}$ (neat) $1682 \mathrm{~cm}^{-1}$.

4-Nitrobenzaldehyde. According to the general procedure 4-nitrobenzyl alcohol provided $238 \mathrm{mg}(79 \%)$ of the product as a pale yellow solid after chromatography (ethyl acetate/hexane=1:19). M.p.: $105{ }^{\circ} \mathrm{C} ;{ }^{1} \mathrm{H}$ NMR $\left(\mathrm{CDCl}_{3}, 300 \mathrm{MHz}\right) \delta 10.3(\mathrm{~s}, 1 \mathrm{H}), 8.3(\mathrm{~d}, 2 \mathrm{H}, J=8.3 \mathrm{~Hz}), 8.0(\mathrm{~d}, 2 \mathrm{H}, J=8.3 \mathrm{~Hz}) ;{ }^{13} \mathrm{C} \mathrm{NMR}\left(\mathrm{CDCl}_{3}, 75.5\right.$ MHz) $\delta 190.2,151.1,140.1,130.4,124.3 ; \mathrm{IR}(\mathrm{KBr}) 1678 \mathrm{~cm}^{-1}$.

3,4,5-Trimethoxybenzaldehyde. According to the general procedure 3,4,5-trimethoxybenzyl alcohol provided $368 \mathrm{mg}(94 \%)$ of the product as a pale yellow solid after chromatography (ethyl acetate/hexane=1:9). M.p. $74{ }^{\circ} \mathrm{C} ;{ }^{1} \mathrm{H}$ NMR $\left(\mathrm{CDCl}_{3}, 300 \mathrm{MHz}\right) \delta 9.9(\mathrm{~s}, 1 \mathrm{H}), 7.1(\mathrm{~s}, 2 \mathrm{H}), 3.9(\mathrm{~s}, 9 \mathrm{H}) ;{ }^{13} \mathrm{C} \mathrm{NMR}\left(\mathrm{CDCl}_{3}, 75.5\right.$ MHz) $\delta 190.9,153.6,143.7,131.7,106.8,60.9,56.3 ; \mathrm{IR}(\mathrm{KBr}) 1682 \mathrm{~cm}^{-1}$. 
Cinnamaldehyde. According to the general procedure cinnamyl alcohol provided $230 \mathrm{mg}(87 \%)$ of the product as a yellow liquid after chromatography (diethyl ether/hexane=1:9). ${ }^{1} \mathrm{H} \mathrm{NMR}\left(\mathrm{CDCl}_{3}, 300 \mathrm{MHz}\right)$ $\delta 9.7(\mathrm{~d}, 1 \mathrm{H}, J=7.7 \mathrm{~Hz}), 7.4-7.5(\mathrm{~m}, 5 \mathrm{H}), 6.5-6.7(\mathrm{~m}, 2 \mathrm{H}) ;{ }^{13} \mathrm{C} \mathrm{NMR}\left(\mathrm{CDCl}_{3}, 75.5 \mathrm{MHz}\right) \delta 193.4,152.6$, 133.8, 131.1, 129.0, 128.4, 128.3; IR (neat) $1681 \mathrm{~cm}^{-1}$.

Geranial. According to the general procedure geraniol afforded $246 \mathrm{mg}(81 \%)$ of the product as a colorless liquid after chromatography (diethyl ether/hexane=1:9). ${ }^{1} \mathrm{H} \mathrm{NMR}\left(\mathrm{CDCl}_{3}, 300 \mathrm{MHz}\right) \delta 9.9(\mathrm{~d}, 1 \mathrm{H}, J=8.1$ $\mathrm{Hz}), 5.8(\mathrm{~d}, 1 \mathrm{H}, J=8.1 \mathrm{~Hz}), 4.8-4.9(\mathrm{~m}, 1 \mathrm{H}), 2.1-2.2(\mathrm{~m}, 4 \mathrm{H}), 1.9(\mathrm{~s}, 3 \mathrm{H}), 1.7(\mathrm{~s}, 3 \mathrm{H}), 1.6(\mathrm{~s}, 3 \mathrm{H}) ;{ }^{13} \mathrm{C}$ NMR $\left(\mathrm{CDCl}_{3}, 75.5 \mathrm{MHz}\right) \delta 191.0,163.5,132.9,127.2,122.4,40.5,25.6,25.5,17.5,17.4$; IR (neat) 1678 $\mathrm{cm}^{-1}$.

Heptanal. The oxidation was performed as described in the general procedure using $580 \mathrm{mg}$ (5 mmol) of 1heptanol and the product was obtained $228 \mathrm{mg}(40 \%)$ as a colorless liquid after distillation. ${ }^{1} \mathrm{H} \mathrm{NMR}\left(\mathrm{CDCl}_{3}\right.$, $300 \mathrm{MHz}) \delta 9.8(\mathrm{t}, 1 \mathrm{H}, J=7.3 \mathrm{~Hz}), 2.4(\mathrm{~m}, 2 \mathrm{H}), 1.1-1.6(\mathrm{~m}, 8 \mathrm{H}), 0.9(\mathrm{t}, 3 \mathrm{H}, J=6.0 \mathrm{~Hz}) ;{ }^{13} \mathrm{C} \mathrm{NMR}$ $\left(\mathrm{CDCl}_{3}, 75.5 \mathrm{MHz}\right) \delta 202.7,43.9,31.6,28.8,22.5,22.1,13.9 ;$ IR (neat) $1724 \mathrm{~cm}^{-1}$.

Decanal. The oxidation was performed as described in the general procedure using $630 \mathrm{mg}(4 \mathrm{mmol})$ of decanol and the product was obtained in $237 \mathrm{mg}(38 \%)$ as a colorless liquid after distillation. ${ }^{1} \mathrm{H}$ NMR $\left(\mathrm{CDCl}_{3}, 300 \mathrm{MHz}\right) \delta 9.9(\mathrm{t}, 1 \mathrm{H}, J=7.3 \mathrm{~Hz}), 2.2-2.4(\mathrm{~m}, 2 \mathrm{H}), 1.2-1.7(\mathrm{~m}, 14 \mathrm{H}), 0.9(\mathrm{t}, 3 \mathrm{H}, J=6.0 \mathrm{~Hz}) ;{ }^{13} \mathrm{C}$ NMR $\left(\mathrm{CDCl}_{3}, 75.5 \mathrm{MHz}\right) \delta$ 202.5, 43.7, 31.7, 29.6, 29.5, 29.4, 29.3, 22.5, 22.1, 14.5; IR (neat) $1716 \mathrm{~cm}^{-}$ 1.

Furfural. According to the general procedure furfurol gave $163 \mathrm{mg}(85 \%)$ of the product as a colorless liquid after chromatography (diethyl ether/hexane=1:9). ${ }^{1} \mathrm{H} \mathrm{NMR}\left(\mathrm{CDCl}_{3}, 300 \mathrm{MHz}\right) \delta 9.7(\mathrm{~s}, 1 \mathrm{H}), 7.7(\mathrm{~s}, 1 \mathrm{H}), 7.3$ (s, 1H), $6.6(\mathrm{~s}, 1 \mathrm{H}) ;{ }^{13} \mathrm{C} \mathrm{NMR}\left(\mathrm{CDCl}_{3}, 75.5 \mathrm{MHz}\right) \delta 177.8,152.9,148.1,121.1,112.6$; IR (neat) $1681 \mathrm{~cm}^{-}$ 1 
2-Pyridinecarboxaldehyde. According to the general procedure pyridine-2-methanol gave $171 \mathrm{mg}(80 \%)$ of the product as a pale yellow liquid after chromatography (ethyl acetate/hexane=1:9). ${ }^{1} \mathrm{H} \mathrm{NMR}\left(\mathrm{CDCl}_{3}, 300\right.$ $\mathrm{MHz}) \delta 10.3(\mathrm{~s}, 1 \mathrm{H}), 8.7-8.8(\mathrm{~m}, 1 \mathrm{H}), 7.8-8.0(\mathrm{~m}, 2 \mathrm{H}), 7.5-7.6(\mathrm{~m}, 1 \mathrm{H}) ;{ }^{13} \mathrm{C} \mathrm{NMR}\left(\mathrm{CDCl}_{3}, 75.5 \mathrm{MHz}\right)$ $\delta 193.3,152.7,150.2,137.0,127.8,121.6$; IR (neat) $1716 \mathrm{~cm}^{-1}$.

Acetophenone. The oxidation was performed as described in the general procedure using $1.2 \mathrm{~g}(10 \mathrm{mmol}) \mathrm{of}$ 1-phenylethanol and the product was obtained in $1.0 \mathrm{~g}(85 \%)$ as a colorless liquid after distillation. ${ }^{1} \mathrm{H}$ NMR $\left(\mathrm{CDCl}_{3}, 300 \mathrm{MHz}\right) \delta 7.8-8.0(\mathrm{~m}, 2 \mathrm{H}), 7.3-7.6(\mathrm{~m}, 3 \mathrm{H}), 2.6(\mathrm{~s}, 3 \mathrm{H}) ;{ }^{13} \mathrm{C} \mathrm{NMR}\left(\mathrm{CDCl}_{3}, 75.5 \mathrm{MHz}\right) \delta 197.9$, 137.2, 133.0, 128.5, 128.2, 26.5; IR (film) $1683 \mathrm{~cm}^{-1}$.

Benzophenone. According to the general procedure diphenylmethanol gave $343 \mathrm{mg}(94 \%)$ of the product as a colorless solid after chromatography (diethyl ether/hexane=1:9). M.p.: $48{ }^{\circ} \mathrm{C} ;{ }^{1} \mathrm{H} \mathrm{NMR}\left(\mathrm{CDCl}_{3}, 300 \mathrm{MHz}\right) \delta$ 7.7-7.8 (m, 4H), 7.4-7.6 (m, 6H); ${ }^{13} \mathrm{C} \mathrm{NMR}\left(\mathrm{CDCl}_{3}, 75.5 \mathrm{MHz}\right) \delta 196.6,137.5,132.3,129.9,128.2$; IR (KBr) $1661 \mathrm{~cm}^{-1}$.

Cyclohexanone. The oxidation was performed as described in the general procedure using $990 \mathrm{mg}$ (10 mmol) of cyclohexanol and the product was obtained in $735 \mathrm{mg}(75 \%)$ as a colorless liquid after distillation. ${ }^{1} \mathrm{H}$ NMR $\left(\mathrm{CDCl}_{3}, 300 \mathrm{MHz}\right) \delta 2.3-2.4(\mathrm{~m}, 4 \mathrm{H}), 1.7-1.9(\mathrm{~m}, 6 \mathrm{H}) ;{ }^{13} \mathrm{C} \mathrm{NMR}\left(\mathrm{CDCl}_{3}, 75.5 \mathrm{MHz}\right) \delta 211.7,41.9,27.1$, $25.0 ;$ IR (neat) $1717 \mathrm{~cm}^{-1}$.

$( \pm)$-Menthone. According to the general procedure $( \pm)$-menthol gave $238 \mathrm{mg}(77 \%)$ of the product as a colorless liquid after chromatography (diethyl ether/hexane=1:9). ${ }^{1} \mathrm{H} \mathrm{NMR}\left(\mathrm{CDCl}_{3}, 300 \mathrm{MHz}\right) \delta 2.2-2.4(\mathrm{~m}$, 1H), 1.7-2.1 (m, 6H), 1.4-1.6 (m, 2H), 0.7-1.0 (m, 9H); ${ }^{13} \mathrm{C} \mathrm{NMR}\left(\mathrm{CDCl}_{3}, 75.5 \mathrm{MHz}\right) \delta 212.0,55.7,50.8$, $35.3,33.6,27.8,25.9,22.2,21.1,18.7$; IR (neat) $1714 \mathrm{~cm}^{-1}$.

Cyclododecanone. According to the general procedure cyclododecanol gave $195 \mathrm{mg}(81 \%)$ of the product as a colorless solid after chromatography (diethyl ether/hexane=1:9). M.p.: $58{ }^{\circ} \mathrm{C} ;{ }^{1} \mathrm{H} \mathrm{NMR}\left(\mathrm{CDCl}_{3}, 300 \mathrm{MHz}\right)$ $\delta$ 2.2-2.4 (m, 4H), 1.7-2.1 (s, 4H), 1.2-1.7 (m, 14H); IR (KBr) $1710 \mathrm{~cm}^{-1}$. 